Published in final edited form as:

Vox Sang. 2017 August ; 112(6): 598-600. doi:10.1111/vox.12541.

\title{
The impact of vaccination on $\mathrm{RBC}$ alloimmunization in a murine model
}

\author{
Prabitha Natarajan ${ }^{1}$, Manjula Santhanakrishnan ${ }^{1}$, Christopher A. Tormey ${ }^{2,1}$, and Jeanne E. \\ Hendrickson ${ }^{1,3}$ \\ ${ }^{1}$ Yale University School of Medicine, Department of Laboratory Medicine, New Haven, CT \\ ${ }^{2}$ VA CT Healthcare System, Pathology and Laboratory Medicine Service, West Haven, CT \\ ${ }^{3}$ Yale University School of Medicine, Department of Pediatrics, New Haven, CT
}

\section{Summary}

\begin{abstract}
Emerging data in animal models and humans suggest that pathogen-associated and damageassociated molecular patterns variably impact RBC alloantibody formation. In this study, we tested the hypothesis that vaccinations may enhance immune responses to transfused RBCs. The Pneumovax 23 vaccine decreased the magnitude of anti-KEL alloimmunization in a murine model, whereas the hepB vaccine did not impact the response; RBC transfusion did not alter immune responses to either vaccine. These data highlight the complexities of the intersection of innate and adaptive immunity and suggest that future studies investigating the pathways through which inflammation impacts alloimmunization are warranted.
\end{abstract}

\section{Keywords}

vaccination; red blood cell; alloimmunization

\begin{abstract}
Red blood cell alloantibodies, induced in some patients through transfusion or pregnancy, can be clinically significant in both settings. Inflammation has been associated with increased rates of RBC alloimmunization in humans[1,2] and in mouse models[3]. We hypothesized that vaccinations, which may be administered during a clinic visit in close proximity with an RBC transfusion, might provide inflammatory signals capable of driving RBC alloimmunization. We tested this hypothesis in a reductionist murine model, which allows an evaluation of the humoral immune response to a single human blood group protein (KEL) expressed on donor and lacking on recipient RBCs. We selected the Pneumovax23 and the hepatitis $\mathrm{B} /$ hepB (recombivax $\mathrm{HB}$ ) vaccines for study: Pneumovax 23 is an adjuvant free polysaccharide vaccine that acts in a $\mathrm{T}$ cell independent manner, whereas hepB contains alum (potassium aluminum sulfate) and acts in a T-cell dependent manner.
\end{abstract}

\footnotetext{
Corresponding author: Jeanne E. Hendrickson, MD, 330 Cedar Street, Clinic Building 405, PO Box 208035, New Haven, CT 06520-8035; jeanne.hendrickson@yale.edu; phone: (203) 737-8316.

PN and JEH designed the experiments; PN and MS completed the experiments; PN, MS, CT, and JEH interpreted the data; PN and JEH wrote the manuscript and all authors edited the manuscript.

Competing Financial Interests: The authors declare no competing financial interests.
} 
Wild-type (WT) C57BL/6 recipient mice were given an intramuscular injection of Pneumovax 23 or saline, and 3-4 hours later were transfused with 1 human equivalent of packed leukoreduced KEL RBCs (see supporting information for additional methodologic details). All mice that were transfused generated anti-KEL alloantibodies, as measured using a flow cytometric crossmatch assay (Figure 1A). However, the magnitude of the anti-KEL $\mathrm{IgG}$ antibody response was significantly lower in mice vaccinated with Pneumovax 23 as compared to the unvaccinated controls (Figure 1B). To confirm that the vaccinations themselves were immunogenic, we measured levels of anti-Pneumovax $23 \operatorname{IgM}$ antibodies by ELISA 1-week post-vaccination. Regardless of transfusion status, sera from all vaccinated mice had evidence of anti-Pneumovax antibodies (Figure 1C).

We next investigated the effects of the hepB vaccine on KEL alloimmunization. Unlike the blunted anti-KEL responses observed in the Pneumovax 23 group, mice transfused in the presence or absence of the hepB vaccine made equally robust anti-KEL responses (Figure 2A). Independent of transfusion status, all vaccinated mice also had evidence of anti-hepB antibodies (Figure 2B).

Emerging data in murine models[4, 5] and in humans[1] suggest that the type of an adjuvant or the type of infection influences alloimmune responses. Thus, we next investigated the impact of viral-like (polyinosinic polycytidylic acid, poly (I:C)) or bacterial (endotoxin lipopolysaccharide (LPS)) inflammation on KEL alloimmunization. Whereas poly I:C significantly boosted the magnitude of the anti-KEL IgG response in a manner similar to what we have previously observed[6], $50 \mu \mathrm{g}$ of LPS given in the peri-transfusion period did not enhance the response (Figure 2C).

Mechanistic studies of vaccines and their adjuvants[7] highlight the complexities of the intersection of innate and adaptive immunity. The type of adjuvant/antigen combination in a vaccine impacts whether the recipient's immune response will be of the Th1, Th2, or mixed type[8], with alum being the most common adjuvant used in vaccines in the US.

Autoimmune sequelae of vaccination[9] have been more thoroughly studied than alloimmune sequelae, though no significant increases in HLA alloimmunization status have been observed in solid organ transplant recipients receiving the seasonal influenza vaccine $[10,11]$. A recent study of factor VIII alloantibody responses in a murine hemophilia model reported a decrease in factor VIII alloantibody responses following the influenza vaccine; Tcell migration experiments documented immune deviation away from the alloantigen and towards the influenza vaccine[12]. The blunted anti-KEL responses observed in the current study after Pneumovax23 may be due to a similar immune deviation. Notably, no differences in factor VIII alloantibody responses were observed following a different vaccination (MMR)[12], similar to what we observe with the hepB vaccine.

Our experimental design was practical but by no means exhaustive. We selected vaccines that might routinely be given in clinics, with vaccination and transfusion timing that is clinically relevant, with an RBC alloimmunization model that involves a clinically significant antigen. However, we cannot extrapolate the results of this limited study design to all vaccinations, to all vaccination/transfusion timing scenarios, to all blood group 
antigens, or to all patient populations. Future studies of the impact of immunization on alloimmunization (and vice versa) may be informative.

In conclusion, we found that Pneumovax 23 decreased and hepB did not impact the magnitude of anti-KEL alloantibody responses; RBC transfusion did not impact the humoral immune response to either vaccine. Our findings join data emerging from other studies in mice and humans suggesting that adjuvants, pathogen- associated molecular patterns, and damage-associated molecular patterns variably impact RBC alloantibody formation. A better understanding of these variables is desirable, in working towards RBC alloantibody prevention.

\section{Supplementary Material}

Refer to Web version on PubMed Central for supplementary material.

\section{Acknowledgments}

This work was supported in part by NIH/NHLBI (R01 HL126076) to JEH.

\section{References Cited}

1. Evers D, van der Bom JG, Tijmensen J, et al. Red cell alloimmunisation in patients with different types of infections. Br J Haematol. 2016; 175:956-66. [PubMed: 27539877]

2. Ryder AB, Hendrickson JE, Tormey CA. Chronic inflammatory autoimmune disorders are a risk factor for red blood cell alloimmunization. Br J Haematol. 2016; 174:483-5. [PubMed: 26456151]

3. Ryder AB, Zimring JC, Hendrickson JE. Factors Influencing RBC Alloimmunization: Lessons Learned from Murine Models. Transfusion medicine and hemotherapy : offizielles Organ der Deutschen Gesellschaft fur Transfusionsmedizin und Immunhamatologie. 2014; 41:406-19. [PubMed: 25670928]

4. Hendrickson JE, Desmarets M, Deshpande SS, et al. Recipient inflammation affects the frequency and magnitude of immunization to transfused red blood cells. Transfusion. 2006; 46:1526-36. [PubMed: 16965580]

5. Bao W, Yu J, Heck S, et al. Regulatory T-cell status in red cell alloimmunized responder and nonresponder mice. Blood. 2009; 113:5624-7. [PubMed: 19336757]

6. Stowell SR, Girard-Pierce KR, Smith NH, et al. Transfusion of murine red blood cells expressing the human KEL glycoprotein induces clinically significant alloantibodies. Transfusion. 2014:17989. [PubMed: 23621760]

7. Wilson-Welder JH, Torres MP, Kipper MJ, et al. Vaccine adjuvants: current challenges and future approaches. Journal of pharmaceutical sciences. 2009; 98:1278-316. [PubMed: 18704954]

8. Ruiz JT, Lujan L, Blank M, et al. Adjuvants- and vaccines-induced autoimmunity: animal models. Immunol Res. 2016

9. Watad A, Quaresma M, Brown S, et al. Autoimmune/inflammatory syndrome induced by adjuvants (Shoenfeld's syndrome) - An update. Lupus. 2017:961203316686406.

10. Danziger-Isakov L, Cherkassky L, Siegel H, et al. Effects of influenza immunization on humoral and cellular alloreactivity in humans. Transplantation. 2010; 89:838-44. [PubMed: 20179666]

11. Vermeiren $P$, Aubert V, Sugamele R, et al. Influenza vaccination and humoral alloimmunity in solid organ transplant recipients. Transpl Int. 2014; 27:903-8. [PubMed: 24797932]

12. Lai JD, Moorehead PC, Sponagle K, et al. Concurrent influenza vaccination reduces anti-FVIII antibody responses in murine hemophilia A. Blood. 2016; 127:3439-49. [PubMed: 27034428] 
A

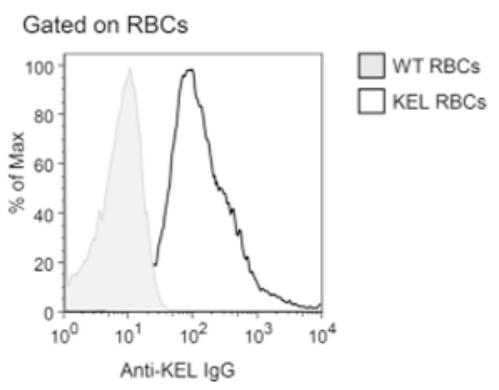

C

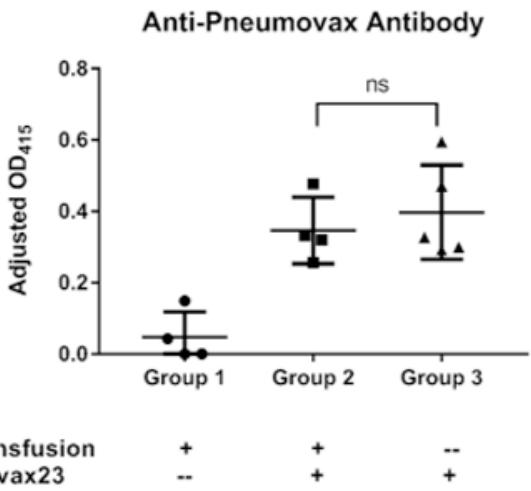

B Anti-KEL Antibody

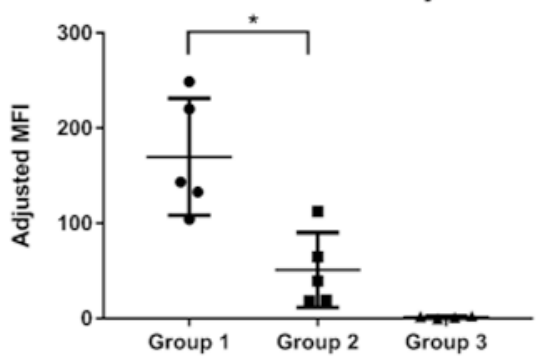

KEL Transfusion

Pneumovax23

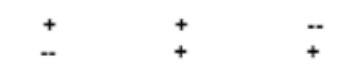

Figure 1. Impact of Pneumovax23 vaccine on KEL alloimmunization

(A) Representative histogram of anti-sera crossmatch. (B) Representative anti-KEL glycoprotein IgG responses 4-weeks post-transfusion, with adjusted mean fluorescence intensity (MFI) being the difference between fluorescence intensity of KEL and WT RBC targets. (C) Representative anti-Pneumovax IgM responses 1-week post vaccination. Data in $B$ and $C$ are representative of two independent experiments ( $n=4$ to 5 mice per group per experiment; each data point represents an animal and bars represent mean $+/-$ s.d.). ${ }^{*} p<0.05$, ns = no significance; determined by Mann Whitney U test. 
A

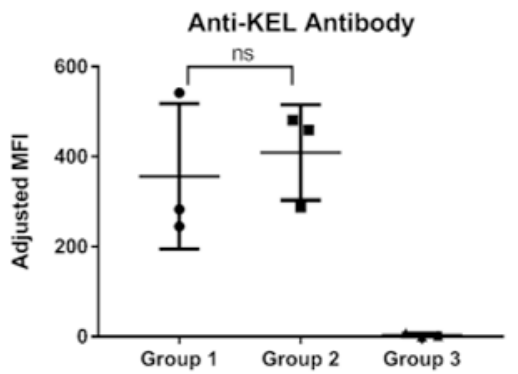

KEL Transfusion HepB Vaccine

C
B

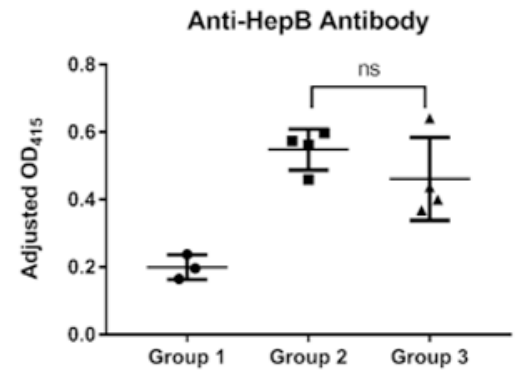
HepB Vaccine

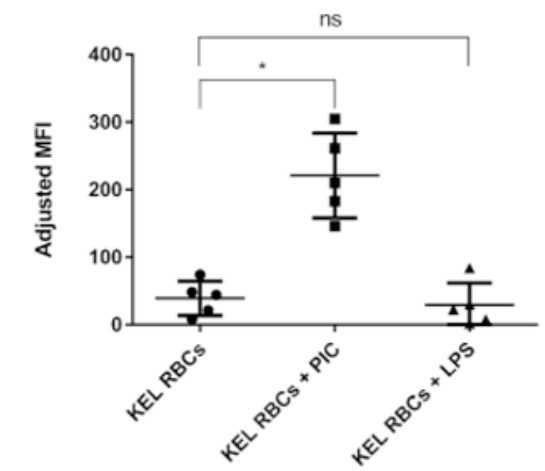

Figure 2. Impact of hepB vaccine or other adjuvants on KEL alloimmunization

(A) Representative anti-KEL glycoprotein IgG responses 4-weeks post transfusion. (B) Representative anti-hepB IgM responses 1-week post- vaccination. (C) Representative antiKEL glycoprotein IgG responses 4-weeks post transfusion, with or without intraperitoneal poly I:C (PIC) or LPS injection. Data in A-C are representative of at least two experiments ( $n=3$ to 5 mice per group per experiment); one pilot experiment showed lower anti-KEL responses in vaccinated animals. Statistics as per Figure 1, but with Kruskal-Wallis with Dunn's Post-test for C. 